\title{
Parálisis cubital aguda producida por pseudoaneurisma de la arteria cubital en el canal de Guyon
}

\author{
F. Albaladejo Mora', P. Sánchez Angulo', M. Hernández Torralba², \\ MV. Redondo Carazo ${ }^{3}$ \\ 'Unidad de Cirugía de la Mano y Microcirugía. Servicio de Cirugía Ortopédica y Traumatología. \\ Hospital General Universitario Reina Sofía. Murcia. España. \\ 2 Servicio de Cirugía Ortopédica y Traumatología. Hospital General Universitario Reina Sofía. Murcia. España. \\ ${ }^{3}$ Servicio de Radiodiagnóstico. Hospital General Universitario Reina Sofía. Murcia. España.
}

Resumen: La parálisis baja del nervio cubital en el canal de Guyon puede producirse por diversas causas entre las que se encuentran los traumatismos agudos o repetitivos, músculos accesorios o lesiones ocupantes de espacio como gangliones, trombosis o pseudoaneurismas, $y$ algunas enfermedades del colágeno.

Para el diagnóstico contamos con métodos no invasivos como la ultrasonografía doppler, la angio-TC y la angio-RM, pero el método de elección es la arteriografía, que dará información precisa del tamaño de la lesión, localización y la presencia o no de circulación colateral.

Presentamos el caso de una paciente de 39 años que sufrió una herida penetrante sobre el canal de Guyon y fue visitada a los I5 días en urgencias con una parálisis cubital aguda. Tras diagnóstico arteriográfico de pseudoaneurisma, y electromiográfico de neurotmesis del nervio cubital, se practicó la resección del mismo y reconstrucción con injerto de vena autólogo invertido, resolviéndose la parálisis de la paciente.

Palabras clave: Pseudoaneurisma, arteria cubital, parálisis cubital, reconstrucción microquirúrgica.

\begin{abstract}
The low ulnar nerve paralysis in the Guyon's canal may be secondary to different causes such us acute o repetitive trauma, accessory muscles or space occupying lesions as ganglion cysts, vascular thrombosis, pseudoaneurysms and collagen diseases.

To make the diagnosis, we have non-invasive imaging techniques such as Doppler sonography, angio-TC and angio-MRI, although the angiography is the gold standard to assess precisely the size, location and the presence or not of collateral circulation.

We present the case of a 39 years-old woman who suffered a penetrating injury of the Guyon's canal who visited the emergency room 15 days after the traumatic episode with an acute ulnar nerve paralysis. The pseudoaneurysm was removed after the arterographic diagnosis and the electromyographic confirmation of neurotmesis. An autologue inverted venous draft was used for reconstruction, and the ulnar nerve paralysis resolved.
\end{abstract}

Key words: Pseudoaneurysm, ulnar artery, ulnar paralysis and microsurgical reconstruction.

Los aneurismas de la extremidad superior se clasifican en verdaderos o falsos, también llamados estos últimos pseudoaneurismas. Los primeros se deben a la lesión de un vaso que permite su dilatación progresiva principalmente después de traumatismos repetitivos como ocurre en la arteria cubital distal y arco superficial'. Los pseudoaneurismas ocurren generalmente tras traumatismos penetrantes o secundarios a fracturas, aplastamientos, etc. ${ }^{2-5}$.

La arteria cubital es la que con mayor frecuencia se ve afectada en el miembro superior por estas lesiones y sobre todo a su paso por el canal de Guyon donde la arteria es más vulnerable a traumatismos y puede ser golpeada por el gancho del ganchoso, como ocurre en ciertos trabajos manuales que predisponen a la aparición de patología vascular por esta característica relación anatómica.
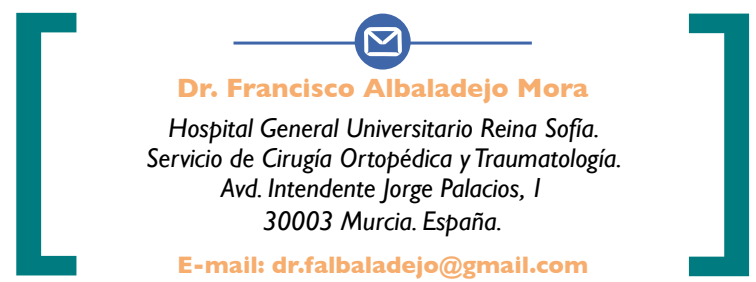
Los aneurismas de la arteria cubital en el canal de Guyon producen el conocido síndrome del martillo hipotenar, descrito en 1772 por Guttani ${ }^{6}$ como una forma poco común de insuficiencia vascular de los dedos que ocurre en sujetos que sobrecargan la parte hipotenar de sus manos, tales como trabajadores manuales, y algunos deportes como las artes marciales, voleyball y baseball7,8.

Los pacientes presentan una masa palpable en la región hipotenar de tamaño variable, pulsátil y que a veces se asocia con signos de embolización distal de los dedos trifalángicos, pero no del pulgar.También y dependiendo de la localización y tamaño del aneurisma pueden aparecer síntomas compresivos del nervio cubital.

Para el diagnóstico contamos con métodos no invasivos como la ultranosografía doppler, la angio-TC y la angio-RM, pero el método de elección es la arteriografía que dará información precisa del tamaño de la lesión, localización y la presencia o no de circulación colateral.

El tratamiento de elección sigue siendo la cirugía con la reconstrucción microquirúrgica del eje arterial ${ }^{9}$ o ligadura de la misma, dependiendo de la circulación colateral y los síntomas del paciente.

Se presenta un caso de pseudoaneurisma de arteria cubital secundario a punción iatrogénica tras sutura de herida simple que presentó síntomas de compresión nerviosa y se trató realizando una resección quirúrgica del mismo y reconstrucción con injerto de vena autólogo invertido.

\section{CASO CLÍNICO}

Paciente mujer de 39 años, que sufre herida incisa en región hipotenar de su mano izquierda, de aproximadamente $1 \mathrm{~cm}$, que suturan en su centro de salud con un punto simple de seda. La sutura es retirada a los 15 días.

Al mes, acude al servicio de urgencias con una masa pulsátil de unos $2 \mathrm{~cm}$ de diámetro sobre canal de Guyon @ Figura I y con clínica de compresión del nervio cubital distal. En la exploración clínica se detecta una hipoestesia en $5^{\circ}$ dedo y región cubital del $4^{\circ}$, con sensibilidad normal en el dorso de la mano. Asimismo se asocia una garra cubital reductible de $4^{\circ}$ y $5^{\circ}$ dedo sin atrofia de la musculatura interósea. (2) Figura 2.

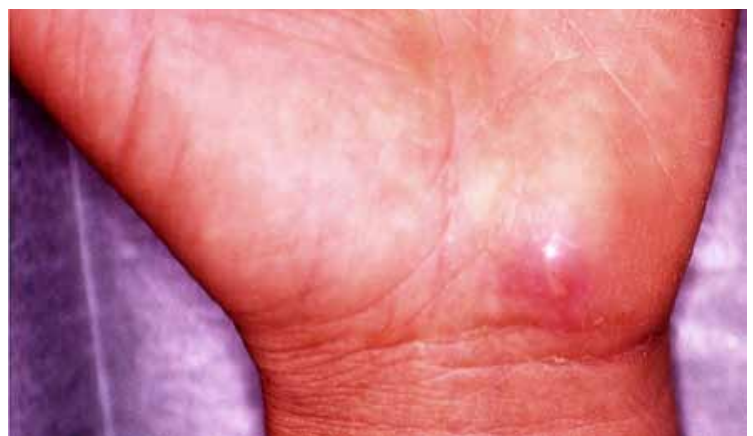

Figura I. Masa pulsátil de $2 \mathrm{~cm}$ de diámetro a nivel del canal de Guyon coincidiendo con la localización de la herida punzante con cristal a los 15 días del traumatismo.

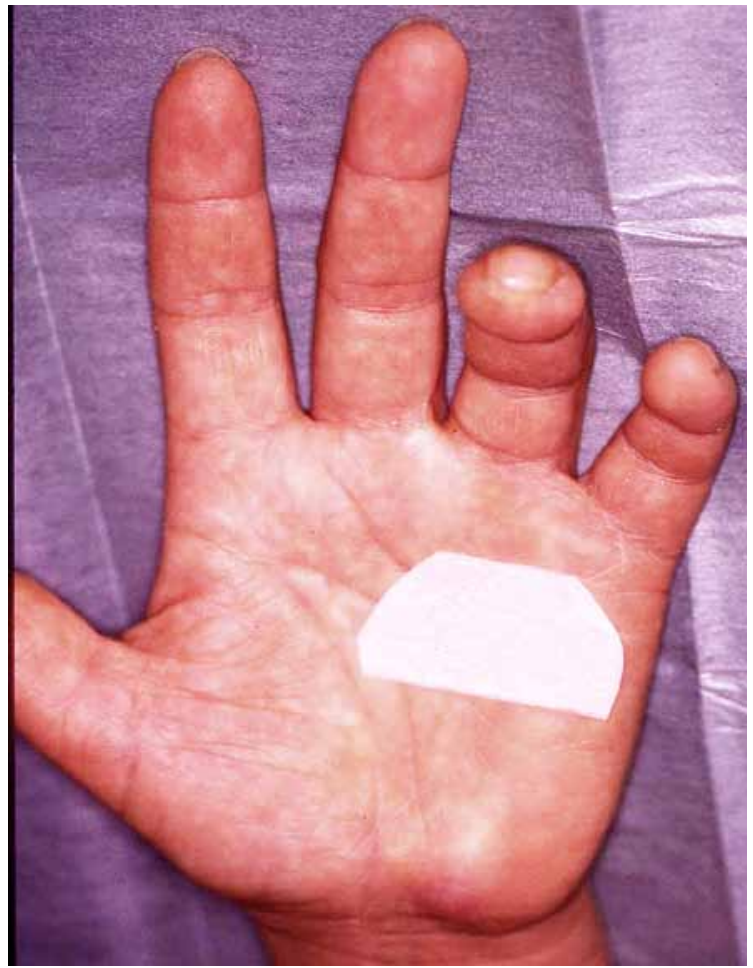

Figura 2. Garra cubital de $4^{\circ}$ y $5^{\circ}$ dedo con imagen de herida incisa en canal de Guyon y masa subyacente.

El test de Froment fue positivo -flexión de la interfalángica del pulgar al sujetar una hoja de papel entre pulgar e índice, por parálisis del músculo adductor pollicis-, así como la prueba de Wartenberg -abducción permanente del $5^{\circ}$ dedo por parálisis del adductor digiti minimi-. No había signos de isquemia digital y tenía buena permeabilidad del colector radial y cubital según la prueba de Allen.

Para su estudio se realizó eco-doppler de la lesión, que mostró dilatación aneurismática de la arteria cubital en 


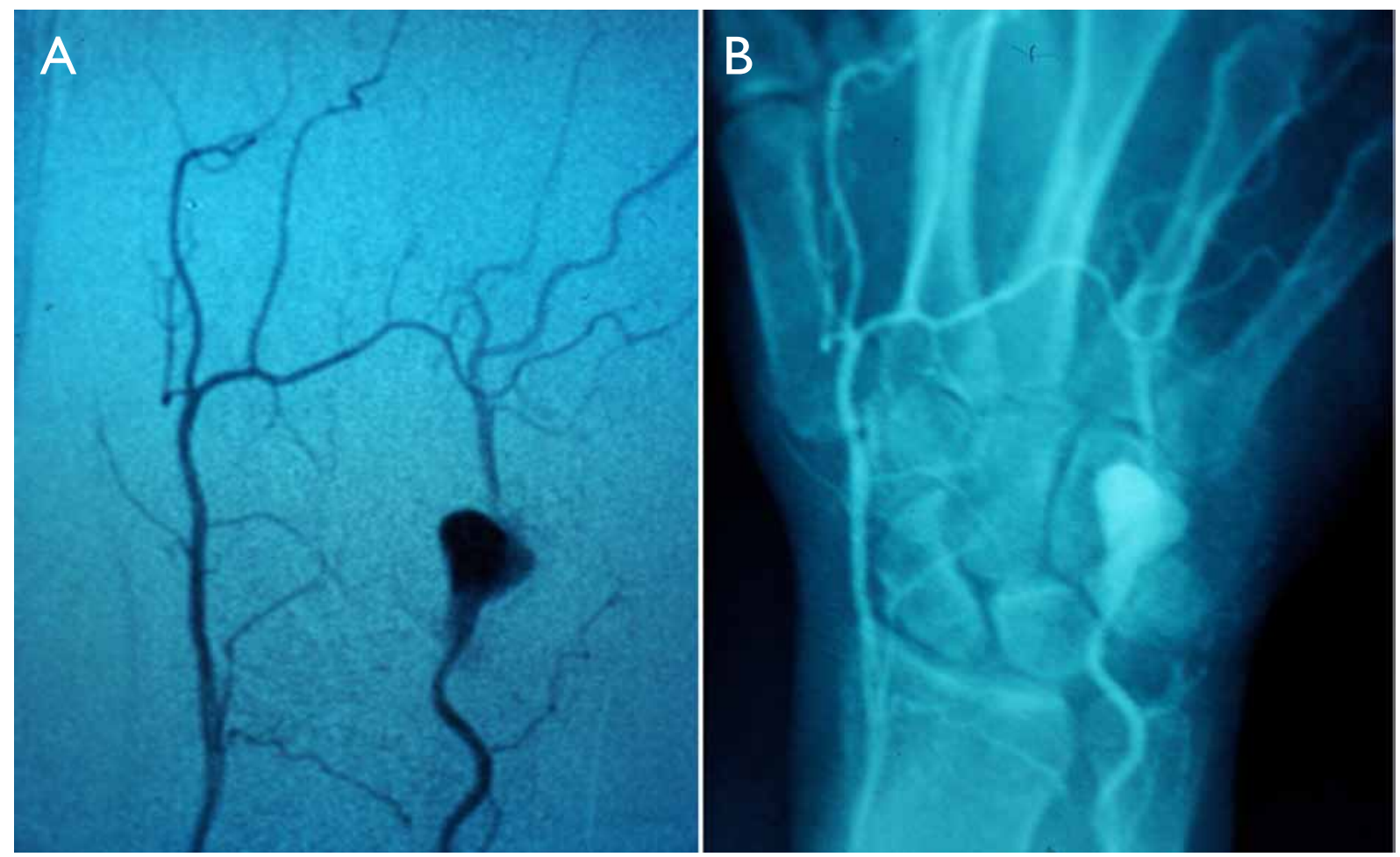

Figura 3. Arteriografía que muestra aneurisma de arteria cubital de $2 \mathrm{~cm}$ de diámetro con buena permeabilidad de arteria cubital y arcos palmares.

el canal de Guyon sin signos de trombosis en su interior y con buena permeabilidad de la luz del vaso.

Para mejor estudio de la lesión y para la planificación quirúrgica se realizó arteriografía que confirmó el diagnóstico ecográfico de aneurisma de aproximadamente $2 \mathrm{~cm}$ de diámetro de arteria cubital en el canal de Guyon con buena permeabilidad de arcos palmares y arterias digitales @ Figura 3.

Se decidió tratamiento quirúrgico con exploración de la zona y reconstrucción vascular con injerto de vena autólogo invertido. Para ello se practicó una incisión amplia longitudinal curvada en el pliegue de flexión de la muñeca que abarcó desde mitad de la palma de la mano con prolongación de unos $10 \mathrm{~cm}$ proximalmente al pliegue de la muñeca (- Figura 4.Tras la disección de los planos subcutáneos y apertura del canal de Guyon se evidenció dilatación aneurismática que comprimía al nervio cubital antes de su división en rama superficial y profunda, como se muestra en la (-) Figura 5. Se procedió a su extirpación y reconstrucción con injerto de vena cefálica tomada de fosa externa del codo mediante incisión transversal siguiendo el pliegue de flexión (- Figura 6. Se colocó el injerto invertido con sutura termino-terminal microquirúrgica con monofi- lamento de 10-0, con ayuda de microscopio 6-40x, e irrigación con suero heparinizado de la luz del vaso y de la sutura.

Tras un postoperatorio satisfactorio desaparecieron los síntomas de compresión nerviosa recuperando la sensibilidad, normalizándose los test de Froment y Wartemberg y desapareciendo la deformidad en garra cubital.

\section{DISCUSIÓN}

Los aneurismas del miembro superior pueden clasificarse en falsos y verdaderos y su etiología puede verse en la (- Tabla I. Los pseudoaneurismas se producen por la penetración de la pared vascular y la consiguiente hemorragia y extravasación sanguínea. El hematoma se organiza y adquiere una cápsula fibrosa que se recanaliza en continuidad con la luz del vaso y se diferencia de ella por la ausencia de revestimiento endotelial.

Éstos son frecuentes tras traumatismos penetrantes accidentales o iatrogénicos. Los aneurismas verdaderos se deben a la lesión de un vaso que permite su dilata- 


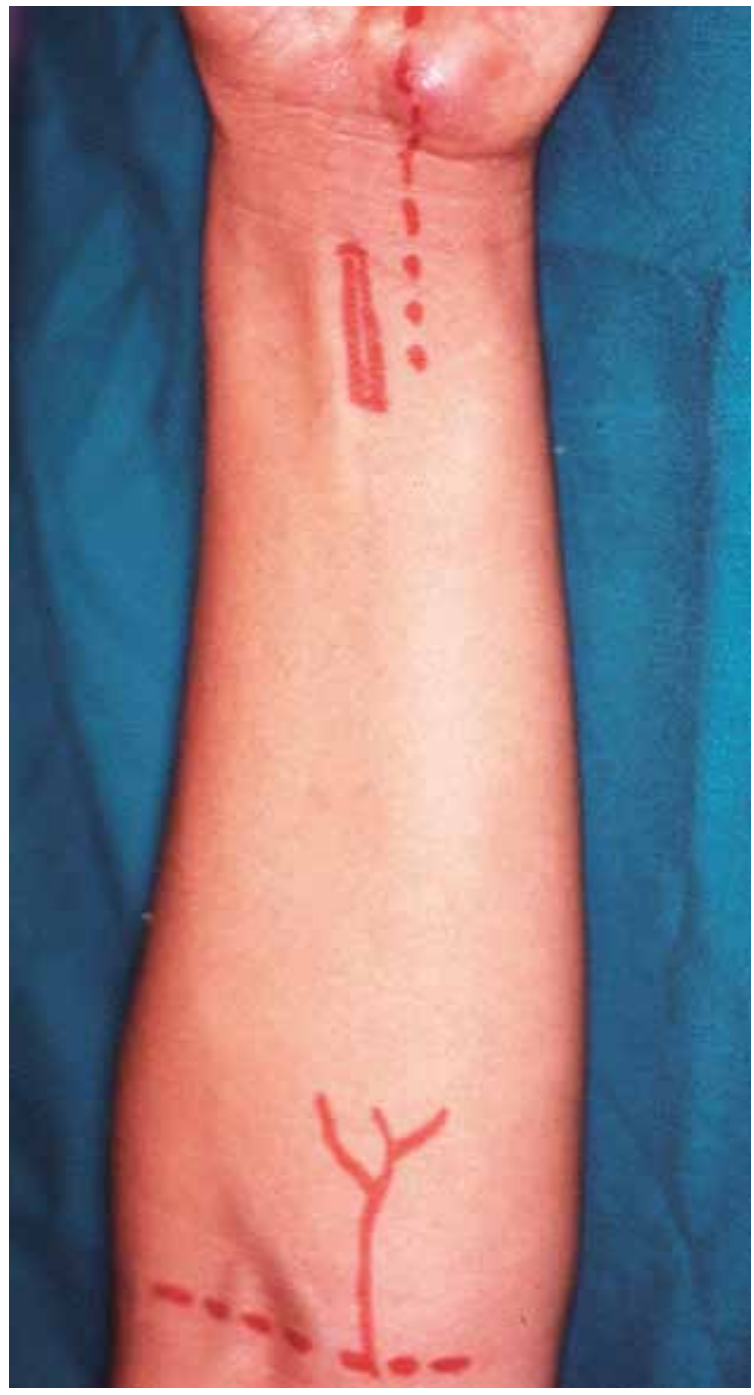

Figura 4. Planificación quirúrgica. Incisiones en muñeca para exploración de canal de Guyon y en codo para toma de injerto de vena cefálica.

\begin{tabular}{c|l} 
VERDADEROS & FALSOS (SEUDOANERIMAS) \\
\hline ARTERIOSCLEROSIS & $\begin{array}{l}\text { PENETRANTES: } \\
\text { Heridas por arma blanca } \\
\text { Cateterismo } \\
\text { Latrógenos: } \\
\text { Bloqueos anestésicos } \\
\text { Cirugia }\end{array}$ \\
\hline CONGÉNITOS & $\begin{array}{l}\text { No penetrantes } \\
\text { Fracturas } \\
\text { Aplastamiento } \\
\text { Infecciones micóticas }\end{array}$ \\
\hline ENF. METABÓLICAS & \\
\hline $\begin{array}{c}\text { ENF. DEL COLÁGENO: } \\
\text { Osteogénesis imperfecta } \\
\text { Enf. Kawasaki } \\
\text { Enf. Buerger }\end{array}$ & \\
\hline Hemofilia & \\
\hline
\end{tabular}

Tabla I. Clasificación de los aneurismas en verdaderos, falsos o iatrogénicos

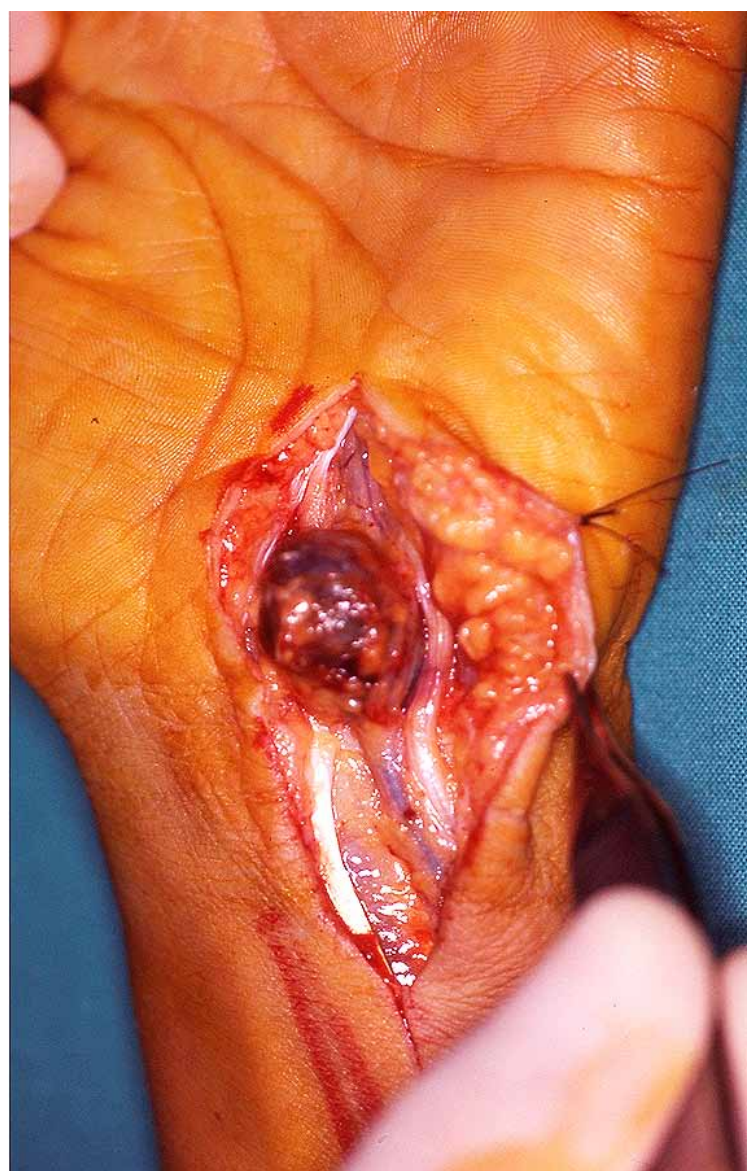

Figura 5. Exploración de canal de Guyon donde se evidencia dilatación aneurismática de arteria cubital comprimiendo al nervio pero con integridad de éste.

ción progresiva y poseen las tres capas de la pared (endotelio, capa muscular y adventicia). Se producen sobre arterias con alteración de la pared como en enfermedades metabólicas y del colágeno, entre otros ${ }^{10,11}$.

Su incidencia está en torno al 1,6\% según Ferris y cols.; aunque la incidencia real es difícil de determinar debido a que muchos casos son asintomáticos. Ocurre con mayor frecuencia en varones (9:1), se asocia con el consumo de tabaco y suele afectar predominantemente a la mano derecha. Marie y cols., presentaron $47 \mathrm{ca}-$ sos de síndrome del martillo hipotenar con un 23,4\% de casos con afectación del nervio cubital. En su estudio, un $91,5 \%$ de los pacientes estuvieron expuestos a traumas repetitivos en la palma como consecuencia de su trabajo, con predominancia en varones $(93,6 \%)$ y una mediana de edad de 42,5 años. El $89 \%$ de los pacientes eran fumadores. En este estudio, un 6,4\% de los pacientes, los síntomas estaban relacionados con traumatismo directo de la eminencia hipotenar'2. 


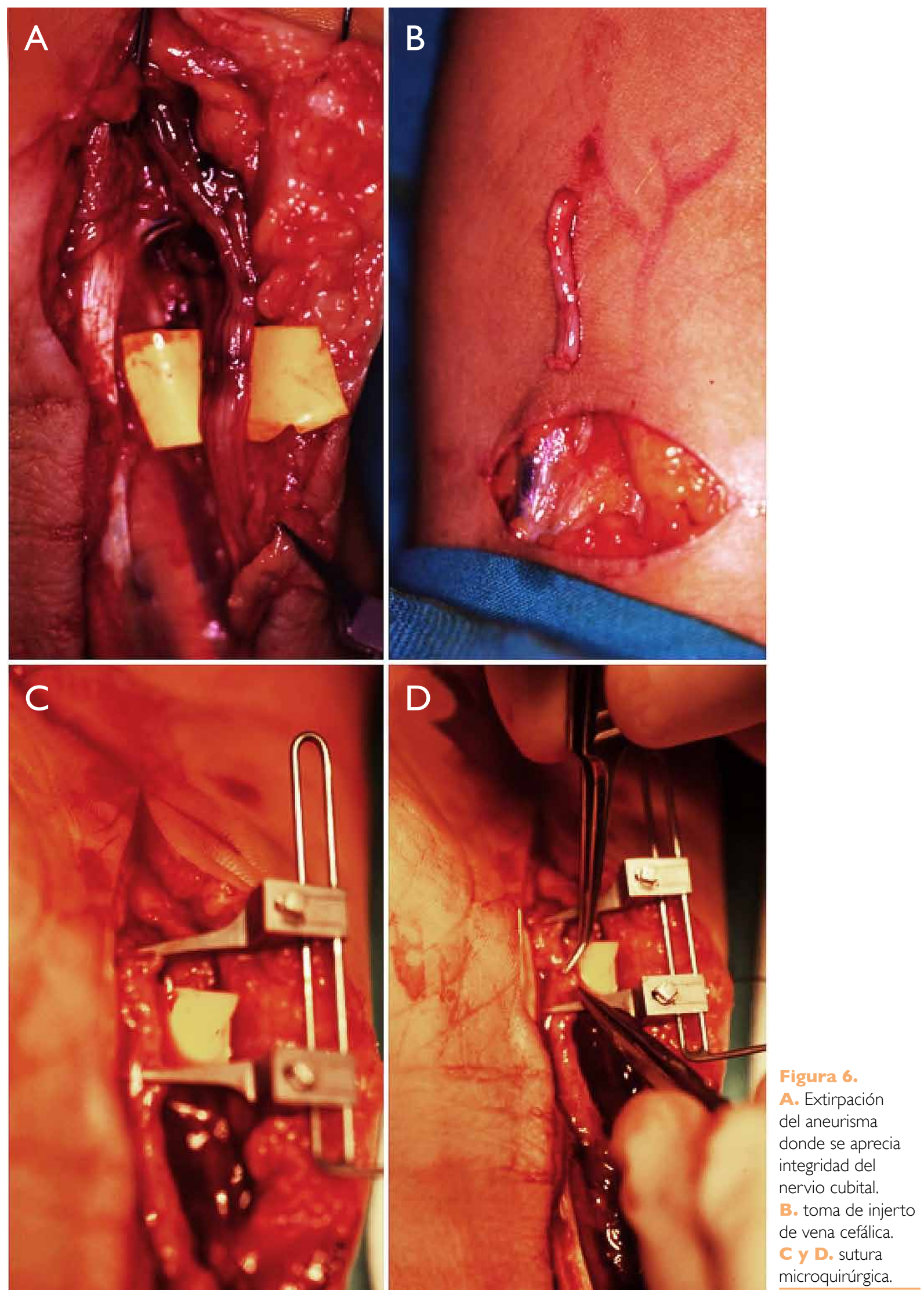


Las manifestaciones clínicas dependen de la extensión de la lesión y la presencia o no de circulación colateral teniendo en cuenta las variaciones anatómicas interindividuales que pueden enmascarar una oclusión arterial. Los síntomas más habituales son una masa palpable y pulsátil en la región hipotenar de tamaño variable, que puede presentar signos de embolización distal de los dedos trifalángicos pero no del pulgar. La presencia de un traumatismo penetrante o traumatismo repetitivos que derivan en la percusión del gancho del ganchoso sobre la pared de la arteria cubital, y aparición de dilatación aneurismática con fenómenos de trombosis, o no, en su interior, que conllevará la presencia de embolias a los dedos largos a través del arco palmar superficial. Puede aparecer dolor, cianosis, palidez cutánea, intolerancia al frío e incluso episodios de vasoespasmo (fenómeno de Raynaud). Si la embolia no se resuelve pueden aparecer ulceración y necrosis digitales.

La dilatación también puede producir fenómenos compresivos del nervio cubital debido a la estrecha relación entre éste y la arteria cubital en un canal estrecho y poco distensible. Los síntomas sensitivos que se evidenciarán serán hipoestesia y parestesias en $5^{\circ}$ dedo y mitad cubital del $4^{\circ}$, sin afectación del lado cubital del dorso de la mano, ya que la rama dorsal del nervio surge antes de entrar en el canal, sirviéndonos este hecho para descartar compresión del nervio en niveles más altos. Los síntomas motores se ponen de manifiesto por la parálisis de los músculos intrínsecos con presencia de garra cubital y atrofia de los espacios interóseos dependiendo del tiempo de evolución del cuadro. Asimismo podemos poner de manifiesto la parálisis del adductor pollicis mediante el signo de Froment, y la parálisis del adductor digiti minimi mediante al signo de Wartenberg.

Para el diagnóstico contamos con métodos no invasivos como la angio-TC, la angio-RM y la ultrasonografía doppler, siendo esta última la primera a realizar por su inocuidad, bajo coste, no utilizar contraste, y por estar ampliamente distribuida en la mayoría de los hospitales $^{13,14}$. Obtendremos información de la forma, localización de la lesión así como de presencia de flujo o no en su interior. Pero el método de elección sigue siendo la arteriografía, aunque sea un método invasivo con uso de contraste ya que además de la forma, tamaño y localización de la lesión, valora la presencia de circulación colateral".

La dilatación aneurismática provoca turbulencias del flujo sanguíneo, trombosis, recanalización y embolias. Debido a la asociación entre aneurismas y trombosis se aconseja el tratamiento quirúrgico tanto de los verdaderos como de los falsos. Entre las opciones terapéuticas tenemos la resección y ligadura, la escisión de la pared dañada e injerto con parche, la resección y la reparación término-terminal y la resección con injerto de interposición. La elección de una técnica u otra depende de la presencia de circulación colateral, así, si ésta es insuficiente para proporcionar una perfusión digital pulsátil o cuando existen fenómenos oclusivos o embólicos se optará por la reconstrucción arterial bien sea mediante sutura término-terminal si se puede hacer sin tensión, lo que ocurrirá generalmente en aneurismas de pequeño tamaño. En cambio, si la circulación colateral es capaz de mantener una buena perfusión digital en ausencia de fenómenos trombo-embólicos se puede optar por la resección del aneurisma y ligadura de los cabos de la arteria. Actualmente, el tratamiento trombolítico está relativamente contraindicado como tratamiento definitivo en los aneurismas de la extremidad superior ${ }^{2,4,8,15}$.

\section{CONFLICTOS DE INTERESES}

Los autores declaran no tener conflictos de intereses.

\section{BIBLIOGRAFÍA}

I. Zimmerman NB. Occlusive vascular disorders of the upper extremity. Hand Clin, 1993; 9: 139-50.

2. McClinton MA. Reconstrution of ulnar artery aneurisma at the wirst. J Hand Surg Am. 20 I I; 36: 328-32.

3. Erdil N, Colak C, Donmez K, Cihan H, Battaloglu B. Pseudoaneurysm of high origin ulnar artery after penetrating trauma. Vasc Endovascular Surg. 20 I0; 44: 609- 12.

4. Yuen JC, Wright E, Johnson LA, Culp WC. Hypothenar hammer syndrome: an update with algorithms for diagnosis and treatment. Ann Plast Surg. 201 1; 67: 429-38.

5. Sanchez A, Archer S, Levine NS, Buchanan RT.Traumatic aneurysm of a common digital artery - a case report. J Hand Surg Am. 1982; 7: 61 9-20.

6. Guttani C. De externis aneurysmatibus manu chirugica methodice pertractandis, 1772. Citado en: Erichsen Jê, trans-ed. Observations on Aneurysm. London: Sydenham Society, 1884: 31 6-8.

7. Abdel-Gawad EA, Bonatti H, Housseini AM, Maged IM, Morgan RF, Hagsplel KD. Hypothenar hammer syndrome in a computer programmer: CTA di- 
agnosis and surgical and endovascular treatment. Vasc Endovascular Surg, 2009; 43: 509- 12.

8. Kaji H, Honma H, Usui M, Yasuno Y, Saito K. Hypothenar hammer syndrome in workers occupationally exposed to vibrating tools. J Hand Surg Br. 1993; 18: 76।-6.

9. Kubo N, Murase T, Moritomo H, Yoshikawa H. Giant aneurysm of the ulnar artery in the palm treated by resection and microvascular reconstruction. Scand J Plast Reconstr Surg Hand Surg. 2009; 43: I $13-6$.

10. Ferris BL, Taylor LM, Oyama K, McLafferty RB, Edwards JM, Moneta GL, et al. Hypothenar hammer syndrome: proposed etiology. JVasc Surg. 2000; 31 : 104-13.

I I. Aulicino PL, Hutton PMJ, Du Puy TE. True palmar aneurysms: A case report and literature review. J Hand Surg Am. 1982; 7: 613-6.
12. Marie I, Hervé F, Primard E, Cailleux N, Levesque $H$. Long-term follow-up of hypothenar hammmer syndrome: a series of 47 patiens. Medicine. 2007; 86: 334-43.

13. DiBenedetto MR, Nappi JF, Ruff ME, Lubbers LM. Doppler mapping in hypothenar syndrome: an alternative to angiography. J Hand Surg Am. 1989; I 4: 244-6.

14. Winterer JT, Ghanem N, Roth M, Schaefer $O$, Lehnhard $S$, Thürl $C$, et al. Diagnosis of the hypothenar hammer syndrome by high-resolution contrast-enhanced MR angiography. Eur Radiol. 2002; 12: 2457-62.

15. Lifchez SD, Higgins JP. Long-term results of surgical treatment for hypothenar hammer syndrome. Plast Reconstr Surg. 2009; 124: 21 0-6. 